\title{
Anaplastic thyroid carcinoma with tongue metastases as primary presentation
}

\begin{abstract}
Anaplastic thyroid carcinoma is one of the most aggressive neoplasms with the worst prognosis, systemic metastases occur in $75 \%$ of patients, but Lingual metastases are exceedinglyr are. Were port a 71 year old female presented with a white nodular painful mass on the left lateral side of the tongue, two weeks later a left node appeared on the second level of the neck. Computed tomography revealed a hypodense mass on the left side of the tongue, heterogenous lymph node on the second left level of the neck and a hypodense mass in the left lobe of the thyroid with calcifications. She was admitted for an incisional biopsy of the tongue tumor, and fine-needle aspirationcytology (FNAC) of the node and thyroid tumor. Histopathologic report was a primary anaplastic thyroid carcinoma with tongue and neck metastases. This case is a very unusual presentation of the usual behavior of anaplastic carcinoma.
\end{abstract}

Special Issue - 2018

\author{
María De Lourdes Flores García, Olga \\ Beltrán Rodriguez Cabo \\ Department of Otorhinolaryngology and Head and Neck \\ Surgery, Instituto Nacional de Rehabilitación Luis Guillermo \\ lbarra Ibarra, Mexico
}

\begin{abstract}
Correspondence: María De Lourdes Flores García MD, Department of Otorhinolaryngology and Head and Neck Surgery, Mexico, Instituto Nacional de Rehabilitación Luis Guillermo Ibarra Ibarra, Mexico City, Mexico,Tel +5255 I27 I20548, Email mariluflogar@gmail.com
\end{abstract}

Received: May 18, 2017 | Published: November 15, 2018

\section{Introduction}

Anaplastic thyroid carcinoma is one of the most aggressive neoplasms with the worst prognosis, it has an average survival of 4-12 months from diagnosis. This carcinoma accounts for 1-2\% of all thyroid cancers with a mean age of presentation between 55-65 years, with a female-to-male ratio of 3:1. Clinically, patients present with symptoms of a rapidly enlarging bulky neck mass with secondary symptoms of hoarseness, dysphagia and dyspnea. Extensive extra thyroidal extension is usually noted at time of surgery, complicating complete resection of the mass. Histologically, anaplastic thyroid carcinoma is characterized by pleomorphic cells with a spectrum of morphologies (spindle, giant cell, rhabdoid and squamoid), frequent mitotic figures including atypical forms, necrosis and vascular invasión. ${ }^{1}$ Systemic metastases occur in $75 \%$ of patients and occur most frequentlyin lung (80\%), bone (6-15\%) and brain $(5-13 \%)$; less frequent metastases such as hepatic, cutaneous, gastrointestinal, and cardiac have been described in the literature. Lingual metastases from anaplastic thyroid carcinoma are exceedingly rare, with only one case reportin the international literature and no report for lingual metastases as a primary manifestation. ${ }^{2-9}$

\section{Case report}

A 71 year old female patient with history of chronic smoking, type 2 diabetes mellitus and arterial hypertension, presented with a white nodular painful mass on the left lateral side of the tongue of one month of duration. She had been treated with mouth washes, antibiotics and antimycotics with no improvement. Two weeks later she noticed aslow-growing, non-painful tumoron the left side of the neck. Physical examination revealed limitation of the oral opening dueto pain and a very painful tumor on the Left part of the Tongue fixed to the floor of the mouth (Figure 1). On the neck an enlarged thyroid was palpated with predominance of the left lobe and a second level node of $3 \mathrm{~cm}$, indurated and fixed to the under lyingt issue. Three weeks after the primary evaluation, she presented with increased volume of the tongue tumor, involving submental region and both submandibular triangles with predominance of the left one. Computed tomography showed an hypodense tumor on the left side of the tongue, heterogenouse lymph node on the second left level of the neck and a hypodense mass in the left lobe of the thyroid with calcifications on the inside. She was admitted for an incisional biopsy of the tongue tumor, and fine-needle aspiration cytology (FNAC) of the node and thyroid tumor (Figure 2). Histopathologic report was a primary anaplastic thyroid carcinoma with tongue and neck metastases (Figure 3). The patient was sent to the National Cancer Institute of Mexico, where they only offered palliative care because she rejected any other therapeutic measures.

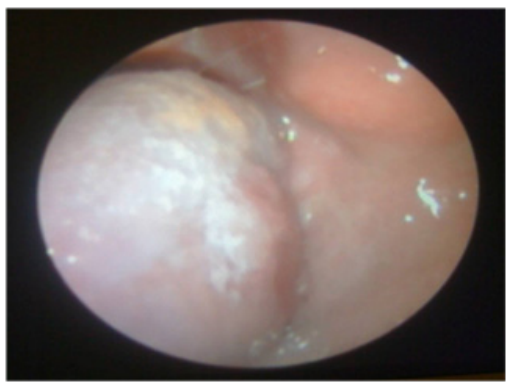

Figure I Left part of the tongue fixed to the floor of the mouth.
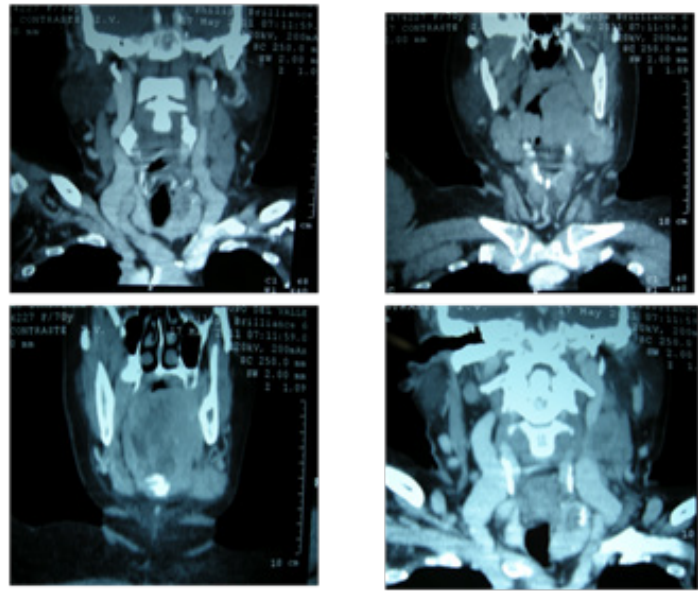

Figure 2 Histopathologic report of thyroid carcinoma with tongue and neck metastases. 

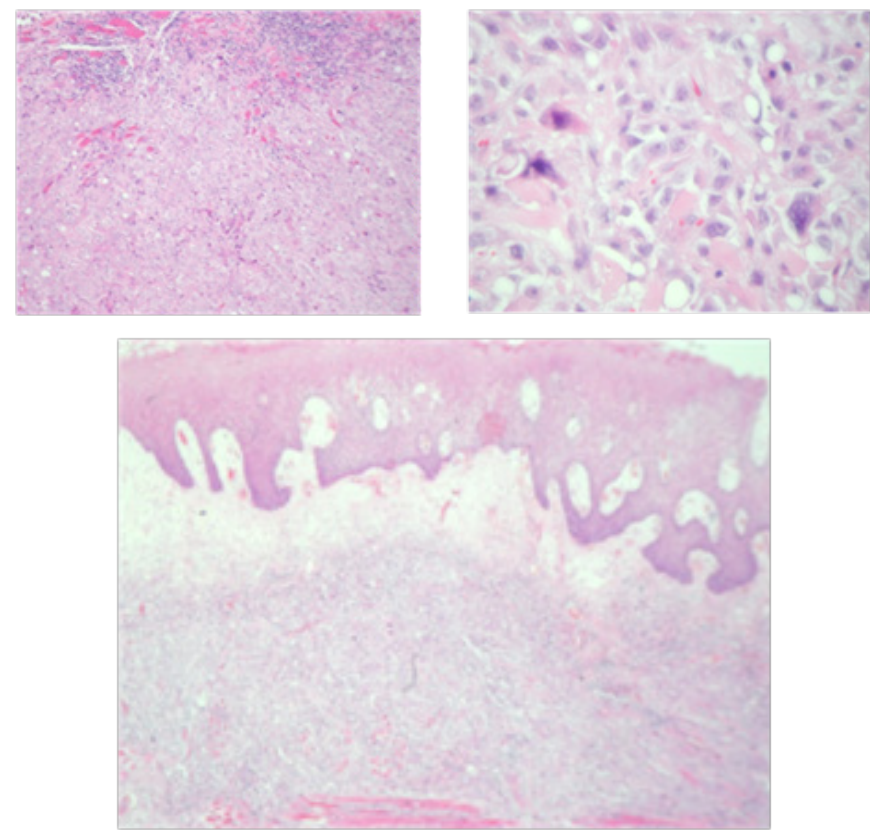

Figure 3 Anaplistic thyroid carcinoma \& lingual metastases of anaplastic thyroid carcinoma.

\section{Discussion}

Anaplastic carcinoma as a very aggressive behavior, that's why it is very rare to debut as a primary manifestation with a distant metastasis. Pomorsky et al. ${ }^{9}$ reported that $2.7 \%$ of 975 patients with thyroid carcinoma presented as primary manifestation with nodular or distant metastasis, however none of them with anaplastic strain. ${ }^{9}$ Because of its aggressiveness, it is common to present distant metastases as a secondary manifestation; however, as mentioned above, the most frequent are lung, bone and brain; there are other sites of less frequent metastes described in the literature, however there's only one reported as a lingual metástasis, ${ }^{8}$ so this would be the second report of anaplastic thyroid carcinoma metastasis to the tongue and the first one a a primary manisfestation.

\section{Conclusion}

This case is a very unusual presentation of the usual behavior of anaplastic carcinoma.

\section{Acknowledgements}

None.

\section{Conflict of interest}

The author declares no conflict of interest.

\section{References}

1. Smallridge, Copland JA. Anaplastic thyroid carcinoma: pathogenesis and emerging therapies. Clin Oncol (R Coll Radiol). 2010;22(6):486-497.

2. Lim, Lee KW, Kim JH, et al. Anaplastic thyroid carcinoma initially presented with abdominal cutaneous mass and hyperthyroidism. Korean J Intern Med. 2010;25(4):450-453.

3. Adamidou, Vlachou M, Haritanti A, et al. Solitary large hepatic metastasis in an elderly patient with anaplastic thyroid carcinoma: a case report. Cases. 2009;J 2(1):41.

4. Nishikawa, Nakashiro K, Sumida T, et al. Mandibular osteoblastic metastasis of poorly differentiated carcinoma of the thyroid gland. Int $J$ Oral Maxillofac Surg. 2010;39(3):292-307.

5. Haas, Celakovský P, Brtková J, et al. Unusual manifestation of anaplastic thyroid cancer. Acta Medica (Hradec Kralove). 2008;51(4):233-236.

6. Dasgupta, Barani IJ, Roach M. Successful radiation treatment of anaplastic thyroid carcinoma metastatic to the right cardiac atrium and ventricle in a pacemaker-dependent patient. Radiat Oncol. 2011;6:16.

7. Besic N, Gazic B. Sites of metastases of anaplastic thyroid carcinoma: autopsy findings in 45 cases from a single Institution. Thyroid. 2013;23(6):709-713

8. Keichi Hori, Motoki Hato, Hiroshi Narita, et al. A case of lingual metastasis of thyroid carcinoma. The journal of the Japanese Practical Surgeon Society. 1995;56(10):2053-2057.

9. Pomorski L, Bartos M. Metastasis as the first sign of thyroid cancer. Neoplasma. 1999;46(5):309-312. 